\title{
Constraining the Accretion Rate Onto Sagittarius A* Using Linear Polarization
}

\author{
Eliot Quataert ${ }^{1}$ and Andrei Gruzinov \\ Institute for Advanced Study, School of Natural Sciences, Einstein Drive, Princeton, NJ 08540; \\ eliot@ias.edu, andrei@ias.edu
}

To appear in the Astrophysical Journal

\begin{abstract}
Two possible explanations for the low luminosity of the supermassive black hole at the center of our galaxy are (1) an accretion rate of order the canonical Bondi value $\left(\sim 10^{-5} M_{\odot} \mathrm{yr}^{-1}\right)$, but a very low radiative efficiency for the accreting gas or $(2)$ an accretion rate much less than the Bondi rate. Both models can explain the broad-band spectrum of the Galactic Center. We show that they can be distinguished using the linear polarization of synchrotron radiation. Accretion at the Bondi rate predicts no linear polarization at any frequency due to Faraday depolarization. Low accretion rate models, on the other hand, have much lower gas densities and magnetic field strengths close to the black hole; polarization may therefore be observable at high frequencies. If confirmed, a recent detection of linear polarization from Sgr $\mathrm{A}^{*}$ at $\gtrsim 150 \mathrm{GHz}$ argues for an accretion rate $\sim 10^{-8} M_{\odot} \mathrm{yr}^{-1}$, much less than the Bondi rate. This test can be applied to other low-luminosity galactic nuclei.
\end{abstract}

Subject Headings: accretion, accretion disks — Galaxy: center — polarization

\section{Introduction}

In this paper we discuss the effect of Faraday depolarization on synchrotron radiation in spherical accretion flow models of low-luminosity galactic nuclei (see also Bower et al 1999ab; Agol 2000). We focus on the radio source Sgr $\mathrm{A}^{*}$ at the Galactic Center, but our results can also be applied to other systems (see $\S 4$ ). This paper was motivated by a possible detection of linear polarization from Sgr A* (Aitken et al. 2000).

The Bondi accretion rate onto the supermassive black hole at the center of our galaxy is estimated to be $\sim 10^{-4}-10^{-5} M_{\odot} \mathrm{yr}^{-1}$ (e.g., Coker \& Melia 1997; Quataert, Narayan, \& Reid 1999), implying a luminosity of $\sim 10^{41} \mathrm{ergs} \mathrm{s}^{-1}$ if the radiative efficiency is $\sim 10 \%$. This is roughly

\footnotetext{
${ }^{1}$ Chandra Fellow
} 
5 orders of magnitudes larger than the observed luminosity (see Narayan et al. 1998 for a recent compilation). Comparable "discrepancies" are obtained for massive elliptical galaxies in nearby X-ray clusters (e.g., Fabian \& Rees 1995; Di Matteo et al. 1999).

One explanation for the low luminosity of nearby supermassive black holes is that they accrete via an advection-dominated accretion flow (ADAF), in which most of the dissipated turbulent energy is stored as thermal energy rather than being radiated (e.g., Rees et al. 1982; Narayan \& Yi 1994, 1995; Abramowicz et al. 1995). In such models the accretion rate is of order the Bondi rate while the radiative efficiency is extremely small $\left(\sim 10^{-6}\right.$ for $\left.\operatorname{Sgr} A^{*}\right)$.

Another explanation for very low luminosity accreting systems is that the Bondi accretion rate estimate is inapplicable (e.g., Blandford \& Begelman 1999; Gruzinov 1999). In particular, numerical simulations of non-radiating accretion flows with small values of the dimensionless viscosity parameter $\alpha$ find that the gas density scales with radius as $\rho \propto r^{-1 / 2}$ rather than the canonical Bondi/ADAF scaling of $\rho \propto r^{-3 / 2}$ (Stone, Pringle, \& Begelman 1999; Igumenshchev \& Abramowicz 1999, 2000; Igumenshchev, Abramowicz, \& Narayan 2000). Narayan, Igumenshchev, \& Abramowicz (2000) and Quataert \& Gruzinov (2000) explained these simulations in terms of a "convection-dominated accretion flow" (CDAF). In such a flow angular momentum is efficiently transported inwards by radial convection, nearly canceling the outward transport by magnetic fields. This strongly suppresses the accretion of matter onto the black hole.

Broad band spectra have thus far had difficulty distinguishing between these explanations for the low luminosity of nearby supermassive black holes. For example, Quataert \& Narayan (1999; hereafter QN) showed that accretion at much less than the Bondi rate could produce spectra quite similar to ADAF models. In this paper we show that linear polarization observations in the radio to sub-mm can provide a sensitive probe of the accretion rate onto the black hole, and help distinguish between degenerate spectral models.

In the next section $(\S 2)$ we present simple estimates of the physical parameters of the accretion flow relevant for our analysis. We then discuss Faraday depolarization in spherical accretion flow models of Sgr $\mathrm{A}^{*}(\S 3)$. In $\S 4$ we compare these predictions with observational constraints on the linear polarization of Sgr $\mathrm{A}^{*}$ and summarize our results. We also generalize our analysis to other low-luminosity galactic nuclei.

Throughout this paper, we focus on accretion models of Sgr A*. An unresolved jet or outflow may, however, dominate the observed emission (e.g., Falcke, Mannheim, \& Biermann 1993; Lo et al. 1998; Falcke 1999); this is briefly discussed in $\S 4$.

\section{Plasma Parameters for Sgr $\mathbf{A}^{*}$}


Stellar kinematics show that there are $\approx 2.6 \times 10^{6} M_{\odot}$ within $\approx 0.015$ pc of the Galactic Center (Eckart \& Genzel 1997, Ghez et al. 1998), centered on the radio source Sgr A* (Menten et al. 1997). The most plausible explanation is that $\mathrm{Sgr} \mathrm{A}^{*}$ is a $\approx 2.6 \times 10^{6} M_{\odot}$ accreting black hole. Sgr $\mathrm{A}^{*}$ is believed to accrete the winds from nearby $(\sim 0.1 \mathrm{pc})$ massive stars (Krabbe et al. 1991). The Bondi accretion rate of these winds onto the supermassive black hole is estimated to be $\approx 10^{-4}-10^{-5} M_{\odot} \mathrm{yr}^{-1}$ (e.g., Coker \& Melia 1997; Quataert, Narayan, \& Reid 1999).

If the accretion rate close to the black hole is of order the Bondi value the gas density near $r \sim 1$ is $n \approx 10^{9}-10^{10} \mathrm{~cm}^{-3}$ (since $v_{r} \approx c$ near the horizon). The corresponding magnetic field strength, assuming rough equipartition with the nearly relativistic protons, is $B \approx 2 \times 10^{3} \mathrm{G}$. At such magnetic field strengths, relativistic electrons cooling by synchrotron radiation would have a cooling time much less than the inflow time of the gas. In order to not overproduce the observed radio to sub-mm luminosity of $\mathrm{Sgr} \mathrm{A}^{*}$, the bulk of the electrons must therefore be marginally relativistic, with $T_{e} \sim 10^{9}-10^{10} \mathrm{~K}$. These plasma parameters $\left(n, B, T_{e}\right)$ describe Bondi and ADAF models of Sgr A* (e.g., Melia 1992, 1994; Narayan, Yi, \& Mahadevan 1995; Narayan et al. 1998). In such models the electrons are assumed to be adiabatically compressed from large radii in the accretion flow, with virtually no additional turbulent heating.

QN showed that accretion at much less than the Bondi rate could also produce the observed high frequency emission from Sgr $\mathrm{A}^{*}$, provided the electrons were much hotter than in standard ADAF models (see their Table 2 and Fig. 8b). A simple explanation for this result can be obtained by applying the Burbidge (1958) estimate to Sgr $\mathrm{A}^{*}$. We consider synchrotron emission from a sphere of radius $R$ containing relativistic electrons with a temperature $k T_{e}=\gamma m_{e} c^{2}$. We take the electron heating rate to be comparable to the net turbulent (magnetic) heating rate. As can be confirmed a posteriori, the synchrotron cooling time is $\gg$ the inflow time of the gas. The electron energy density is then similar to the magnetic energy density

$$
n \gamma m_{e} c^{2} \approx \frac{B^{2}}{8 \pi}
$$

The frequency of peak synchrotron emission and the synchrotron luminosity are given by

$$
\nu \approx 0.1 \gamma^{2} \frac{e B}{m_{e} c}
$$

and

$$
L \approx \sigma_{T} c B^{2} \gamma^{2} R^{3} n
$$

where $\sigma_{T}$ is the Thomson cross section.

We express $n, \gamma$, and $B$ in terms of $R, \nu$, and $L$ (see also Falcke 1996; Beckert \& Duschl 1997)

$$
\begin{gathered}
\gamma \approx 3.2\left(\frac{m_{e}}{c} \frac{\nu^{4} R^{3}}{L}\right)^{1 / 7} \approx 100 \\
n \approx \frac{4}{\gamma^{5} \lambda^{2} r_{e}} \approx 10^{6} \mathrm{~cm}^{-3}
\end{gathered}
$$


and

$$
B \approx \sqrt{8 \pi m_{e} c^{2} \gamma n} \approx 45 \mathrm{G},
$$

where $\lambda=c / \nu$ is the wavelength and $r_{e}=e^{2} /\left(m_{e} c^{2}\right)$ is the classical electron radius. For the numerical estimates in equations (4)-(的), we have used the observed values for Sgr $A^{*}$. The peak synchrotron frequency is at $\nu \approx 10^{3} \mathrm{GHz}$ with a luminosity of $L \approx 10^{36} \mathrm{ergs} \mathrm{s}^{-1}$ (e.g., Serabyn et al. 1997). In spherical accretion models, this high frequency emission arises from very close to the black hole, so we have taken $R \approx R_{g} \approx 10^{12} \mathrm{~cm}$.

Equation (15) gives a density close to the black hole of $n \sim 10^{6} \mathrm{~cm}^{-3}$; the implied accretion rate is then $\sim 10^{-8} M_{\odot} \mathrm{yr}^{-1}$, three to four orders of magnitude smaller than the Bondi value.

The thermal blackbody emission at frequency $\nu$ from a sphere of radius $R$ is

$$
L_{t}=2 \pi \nu^{3} \gamma m_{e} 4 \pi R^{2} \approx 10^{37} \operatorname{ergs~s}^{-1},
$$

where the numerical estimate is for our fiducial parameters. This comparison shows that the synchrotron emission becomes optically thin below the peak frequency, near $\nu \approx 300 \mathrm{GHz}$. At lower frequencies the emission is self-absorbed.

The above considerations show that both low $\left(\sim 10^{-8} M_{\odot} \mathrm{yr}^{-1}\right)$ and high $\left(\sim 10^{-5}-10^{-4} M_{\odot} \mathrm{yr}^{-1}\right)$ accretion rate models can explain the observed sub-mm "bump" in Sgr A*. Such models can be distinguished by comparing the observed brightness temperature and/or radio image as a function of frequency with the theoretical predictions (see, e.g., Özel, Psaltis, \& Narayan 2000). This test has been difficult to implement because interstellar scattering significantly broadens the image of Sgr $A^{*}$. I In the next section we show that the linear polarization of Sgr $\mathrm{A}^{*}$ at high frequencies provides an additional discriminant.

\section{Faraday Depolarization}

The anisotropic index of refraction of a magnetized plasma leads to a frequency-dependent rotation in the position angle, $\theta$, of linearly polarized electromagnetic waves,

$$
\theta=R M \lambda^{2}
$$

where $R M$ is the rotation measure. This can lead to significant depolarization of intrinsically linearly polarized synchrotron emission.

\footnotetext{
${ }^{2}$ Recent detections of Sgr A*'s intrinsic size (Lo et al. 1998; Krichbaum et al. 1998) still have sufficient uncertainties that a range of theoretical models are allowed.
} 
For a "cold" non-relativistic plasma, RM is given by (e.g., Rybicki \& Lightman 1979)

$$
\begin{aligned}
R M & =\frac{e^{3}}{2 \pi m_{e}^{2} c^{4}} \int d \mathbf{l} \cdot \mathbf{B} n \\
& =2.63 \times 10^{-13} \times \int d \mathbf{l} \cdot \mathbf{B} n \frac{\mathrm{rad}}{\mathrm{m}^{2}},
\end{aligned}
$$

where $d \mathbf{l}$ is the differential path length from the observer to the source. In the Appendix we show that the rotation measure for an ultrarelativistic thermal plasma is given by

$$
\begin{aligned}
R M_{\gamma} & =\frac{e^{3}}{2 \pi m_{e}^{2} c^{4}} \int d \mathbf{l} \cdot \mathbf{B} n \frac{\log \gamma}{2 \gamma^{2}} \\
& =2.63 \times 10^{-13} \times \int d \mathbf{l} \cdot \mathbf{B} n \frac{\log \gamma}{2 \gamma^{2}} \frac{\mathrm{rad}}{\mathrm{m}^{2}}
\end{aligned}
$$

where $\gamma=k T_{e} / m_{e} c^{2}$. A comparable expression is obtained for a power law distribution of relativistic electrons, with $\gamma$ replaced by $\gamma_{\text {min }}$, the minimum Lorentz factor of the electrons (Jones \& O'Dell 1977). In what follows, we define $R M(r)$ to be the contribution to the net rotation measure from radii within $d r \approx r$ of radius $r$ in the accretion flow.

\subsection{ADAF/Bondi Models}

In spherical accretion flow models, higher frequency radio emission arises from closer to the black hole, where the electron temperature and magnetic field strengths are the largest; this is also true for jet models (e.g., Falcke 1999). Özel et al. (2000) show that in ADAF models of Sgr A* the synchrotron emission at frequency $\nu=100 \nu_{100} \mathrm{GHz}$ arises from a radius $r_{\nu} \approx 20 \nu_{100}^{-0.9}$ (see their Fig. 5). Th This radius defines the $\tau=1$ surface of the synchrotron emission. For smaller radii the emission is self-absorbed while for larger radii it is optically thin. Faraday rotation is only important for $r \gtrsim r_{\nu}$, where the photons "free stream" out of the accretion flow.

In Bondi/ADAF models Faraday rotation is so strong that the synchrotron emission is completely depolarized by the plasma within $d r \sim r$ of $r_{\nu}$, i.e., in the vicinity of the $\tau=1$ surface where it is emitted (depolarization and emission are thus virtually co-spatial). Taking $n \propto r^{-3 / 2}$ and $B \propto r^{-5 / 4}$; the rotation measure scales roughly as $R M \propto r^{-7 / 4} \gamma^{-2}$. The relativistic suppression of the rotation measure is small in all models which have an accretion rate comparable to the Bondi rate, because the electrons must then be at most marginally relativistic ( 22 ). The rotation measure as a function of radius is thus given by (see also Bower et al. 1999ab)

$$
R M \approx 10^{13} r^{-7 / 4} \mathrm{rad} \mathrm{m}^{-2} .
$$

\footnotetext{
${ }^{3}$ Falcke (1999) finds a similar expression for $r_{\nu}$ in the jet model.
} 
In equation (111) we have assumed that the magnetic field is in rough equipartition with the gas pressure, has a significant component along the line of sight, and has a coherence length $\ell \sim r$; for $\ell \ll r, R M$ is reduced by $\approx(\ell / r)^{1 / 2}$. The normalization in equation (11) is set by the Bondi accretion rate.

The large $R M$ in ADAF/Bondi models leads to a significant rotation in the position angle of linearly polarized waves. Photons of frequency $\nu$ emitted at radius $r_{\nu}$ undergo Faraday rotation through an angle

$$
\theta_{\nu} \approx \lambda^{2} R M\left(r_{\nu}\right) \approx 10^{8} \nu_{100}^{-2} r_{\nu}^{-7 / 4} \sim 10^{6} \nu_{100}^{-0.43} \mathrm{rad},
$$

where the last estimate uses Özel et al.'s (2000) fit to $r_{\nu}(\nu)$.

These rotation angles are so large that the synchrotron emission in ADAF/Bondi models of Sgr $\mathrm{A}^{*}$ is completely depolarized by Faraday rotation. For example, in a simple uniform source model, the observed polarization is $\propto \theta_{\nu}^{-1}$ (Pacholczyk 1970). In general, the observed polarization depends on the rotation measure power spectrum, but is $\ll 1$ for $\theta_{\nu} \gg 1$ (e.g., Tribble 1991). T

\section{2. $\dot{M} \ll \dot{M}_{\text {Bondi }}$}

If the accretion rate onto $\mathrm{Sgr} \mathrm{A}^{*}$ is much less than the Bondi rate, significant polarization may be observable at high frequencies; we show this using an order of magnitude estimate.

For the $\dot{M} \sim 10^{-8} M_{\odot} \mathrm{yr}^{-1}$ model of $\S 2$ the rotation measure calculated using equation (10) is $R M \approx 10^{3} \mathrm{rad} \mathrm{m}^{-2}$ near $r \sim 1$. Moreover, if $n \propto r^{-1 / 2}$, as in CDAF models, the magnetic field scales as $B \propto r^{-3 / 4}$ and

$$
R M \approx 10^{3} r^{-1 / 4}\left(\frac{\gamma}{100}\right)^{-2} \operatorname{rad~} \mathrm{m}^{-2}
$$

The variation of the electron Lorentz factor with radius is somewhat uncertain, but we expect roughly $\gamma \propto r^{-1}$, so that the electrons become non-relativistic by $r \sim 10^{2}$. Equation (13) then shows that RM has its maximal value at large radii, $r \sim 10^{2}$, where $R M \sim 3 \times 10^{6} \mathrm{rad} \mathrm{m}^{-2}$.

Equation (13) demonstrates that, in contrast to ADAF models, there is no depolarization of synchrotron emission at small radii in models with accretion rates much less than the Bondi rate; $R M$ is negligible in the region where the synchrotron emission is produced. Depolarization can still be important, however, because observed photons experience different Faraday rotation at large radii, $r \gtrsim 10^{2}$, on their way out of the accretion flow (e.g., Bower et al. 1999ab).

\footnotetext{
${ }^{4}$ One way of evading this conclusion is to posit that the magnetic field is sufficiently tangled $(\ell \ll r)$ to decrease $R M$ to $\lesssim 10^{6} \mathrm{rad} \mathrm{m}^{-2}$. This tangling would, however, also eliminate linear polarization.
} 
Spatial variation in the rotation measure will depolarize Sgr $\mathrm{A}^{*}$ at frequencies for which $\delta \theta=$ $\lambda^{2} \delta R M \gtrsim \pi$, i.e., for

$$
\nu \lesssim 100\left(\frac{\delta R M}{10^{6} \mathrm{rad} \mathrm{m}^{-2}}\right)^{1 / 2} \mathrm{GHz},
$$

where $\delta R M$ is the difference in the rotation measure for photons of a given frequency which travel through different parts of the accretion flow. Q Quantitative calculations of depolarization by differential Faraday rotation are uncertain; two points are, however, clear: (1) At low frequencies, $\ll 100 \mathrm{GHz}$, Sgr $\mathrm{A}^{*}$ is easily depolarized at $r \gtrsim 10^{2}$. The required $\delta R M$ is $\ll 10^{6} \mathrm{rad} \mathrm{m}^{-2}$, orders of magnitudes smaller than the values of $R M$ obtained at $r \sim 10^{2}-10^{4}$. (2) Emission above $\sim 100$ $\mathrm{GHz}$ can plausibly be linearly polarized if the accretion rate onto $\mathrm{Sgr} \mathrm{A}^{*}$ is much less than the Bondi rate. In particular, equations (13) and (14) show that for $\dot{M} \ll \dot{M}_{\text {Bondi }}$, emission above $\approx 100 \mathrm{GHz}$ is not depolarized propagating out of the accretion flow.

\section{Discussion}

$\mathrm{ADAF} /$ Bondi models assume that the accretion rate onto $\mathrm{Sgr} \mathrm{A}^{*}$ is of order the Bondi rate $\left(\sim 10^{-4}-10^{-5} M_{\odot} \mathrm{yr}^{-1}\right)$ and that the radio to infrared emission is produced by synchrotron emission from marginally relativistic electrons $\left(T_{e} \approx 10^{9}-10^{10} \mathrm{~K}\right)$. In such models the rotation measure is $\gtrsim 10^{10} \mathrm{rad} \mathrm{m}^{-2}$ inside $\approx 100$ Schwarzschild radii where the synchrotron emission is produced. ADAF/Bondi models thus predict that Sgr $\mathrm{A}^{*}$ should be depolarized by Faraday rotation over the entire radio to infrared spectrum, and should have nearly zero linear polarization.

The theoretical arguments summarized in $\S 1$ propose that the accretion rate onto Sgr $\mathrm{A}^{*}$ is much less than the Bondi rate. We have described one such model, in which the electron heating rate is of order the rate of change of the magnetic energy density. For an accretion rate $\sim 10^{3}$ times smaller than the Bondi rate, i.e., $\sim 10^{-8} M_{\odot} \mathrm{yr}^{-1}$, and with relativistic electrons with $\gamma \approx 100$, this model can explain the observed high frequency emission from Sgr $A^{*}$. Moreover, it predicts that the rotation measure in the accretion flow is much smaller than in ADAF/Bondi models. This is because the gas density and magnetic field strength close to the black hole are much smaller, and because the electrons are relativistic $\left(R M \propto \gamma^{-2} \log \gamma\right.$ for $\gamma \gg 1$; see $\S 3$ and the Appendix). The maximal contribution to the rotation measure comes from $\sim 10^{2}-10^{3}$ Schwarzschild radii, where $R M \sim 10^{6} \mathrm{rad} \mathrm{m}^{-2}$.

Rotation measures of $\sim 10^{6} \mathrm{rad} \mathrm{m}^{-2}$ can depolarize Sgr $\mathrm{A}^{*}$ at $\nu \ll 100 \mathrm{GHz}$ by differential Faraday rotation; photons of a given frequency travel through different rotation measures on their

\footnotetext{
${ }^{5}$ To be precise, $\delta R M$ is the difference in the rotation measure at $r \sim 100-10^{4}$ on scales of $r_{\nu}$, the source size at frequency $\nu$. This is difficult to calculate analytically, but could be determined from future MHD simulations of non-radiating accretion flows.
} 
way out of the accretion flow. Following Bower et al. (1999ab), we believe that this accounts for the $\lesssim 0.2 \%$ linear polarization of $\mathrm{Sgr} \mathrm{A}^{*}$ at low frequencies (from $\approx 4$ to $\approx 23 \mathrm{GHz}$; see Bower et al. 1999ab): it is less clear, however, that it can account for Bower et al.'s (1999b) limit of $\lesssim 1 \%$ linear polarization at $86 \mathrm{GHz}$ (see below). In fact, rotation measures of $\approx 10^{6} \mathrm{rad} \mathrm{m}^{-2}$ are insufficient to depolarize emission above $\approx 100 \mathrm{GHz}$. As a result, in models with accretion rates much less than the Bondi rate, $\gtrsim 100 \mathrm{GHz}$ emission is not depolarized propagating out of the accretion flow; intrinsically polarized synchrotron emission may therefore be observable at high frequencies.

The above considerations show that the linear polarization of $\operatorname{Sgr} \mathrm{A}^{*}$ at high frequencies provides a means of distinguishing between accretion at the Bondi rate, and accretion at a much smaller rate. In fact, Aitken et al. (2000) report a possible detection of $\sim 10 \%$ linear polarization from Sgr A* between 150 and $400 \mathrm{GHz}$. If confirmed, these observations require an accretion rate onto Sgr $\mathrm{A}^{*}$ much less than the Bondi rate, roughly $\dot{M} \sim 10^{-8} M_{\odot} \mathrm{yr}^{-1}$.

One difficulty in interpreting Aitken et al's results is the large beam $\left(\approx 20^{\prime \prime}\right)$ of the SCUBA camera on the JCMT. This large beam forced Aitken et al. to subtract out free-free and (polarized!) dust emission in order to isolate the flux and polarization of $\operatorname{Sgr} \mathrm{A}^{*}$. Future high resolution polarimetry at $\mathrm{mm}$ wavelengths is clearly necessary to further address this important issue.

Aitken et al. find that the position angle of Sgr $\mathrm{A}^{*}$ changes by $\lesssim 10^{\circ}$ between $\lambda=0.135 \mathrm{~cm}$ and $\lambda=0.2 \mathrm{~cm}$; at face value this implies $R M \lesssim 10^{5} \mathrm{rad} \mathrm{m}^{-2}$, somewhat smaller than the values of $\sim 10^{6} \mathrm{rad} \mathrm{m}^{-2}$ in our model. This assumes, however, that the intrinsic position angle of Sgr $\mathrm{A}^{*}$ is the same at $\lambda=0.135 \mathrm{~cm}$ and $\lambda=0.2 \mathrm{~cm}$, which need not be the case. Moreover, our estimates of $R M$ are actually upper limits, since they assume (1) equipartition magnetic fields aligned along the line of sight and (2) that our line of sight passes through the equatorial plane of the accretion flow.

Our analysis of depolarization is applicable even if the radio emission from Sgr $\mathrm{A}^{*}$ is dominated by a jet/outflow, rather than the accretion flow as we have assumed. In jet models, it is still natural for the highest frequency emission to originate very close to the black hole; in Falcke's model, e.g., the $\gtrsim 100 \mathrm{GHz}$ emission arises from $\lesssim 10 R_{g}$, in what is really a "transition region" between the accretion flow and the jet (Falcke 1999). In order for this emission to not be depolarized (either in situ or propagating through the accretion flow), our constraints on the rotation measure and the plasma conditions close to the black hole still apply.

Two scenarios in which accretion at the Bondi rate could be consistent with observed linear polarization at high frequencies are (1) if the high frequency emission arises close to the black hole, but in a nearly empty funnel pointed directly towards us (e.g., along the rotation axis of an ADAF) or (2) if the high frequency emission from $\mathrm{Sgr} \mathrm{A}^{*}$ is produced at very large distances from the black

\footnotetext{
${ }^{6}$ Bower et al. (1999ab) showed that "bandwidth" depolarization at $\nu \approx 8 \mathrm{GHz}$ requires $R M>10^{7} \mathrm{rad} \mathrm{m}^{-2}$; this constraint does not, however, apply to the depolarization discussed here, namely that due to a spatially varying $R M$.
} 
hole, $r \gtrsim 10^{3}$. The former possibility requires a rather special geometry and the latter is ruled out by the VLBI source size of $\sim 10 R_{g}$ (Krichbaum et al. 1998) and the variability of Sgr $\mathrm{A}^{*}$ at $\approx 100$ GHz (Tsuboi, Miyazaki, \& Tsutsumi 1999).

\subsection{Application to Other Systems}

Although we have have focused our analysis on Sgr A* at the Galactic Center, linear polarization of high frequency radio emission can be used as a probe of the accretion physics in other low-luminosity galactic nuclei (see, e.g., Nagar et al. 2000 for recent VLA observations of LLAGN). For a black hole of mass $M=m_{9} 10^{9} M_{\odot}$ accreting (spherically) at a rate $\dot{M}=10^{-4} \dot{m}_{-4} \dot{M}_{\text {edd }} \approx$ $10^{23} \dot{m}_{-4} m_{9} \mathrm{~g} \mathrm{~s}^{-1}$, the density, magnetic field strength, and rotation measure in ADAF models are

$$
\begin{gathered}
n \approx 3 \times 10^{6} \dot{m}_{-4} m_{9}^{-1} r^{-3 / 2} \mathrm{~cm}^{-3}, \\
B \approx 100 \dot{m}_{-4}^{1 / 2} m_{9}^{-1 / 2} r^{-5 / 4} \mathrm{G},
\end{gathered}
$$

and

$$
R M \approx 3 \times 10^{10} \dot{m}_{-4}^{3 / 2} m_{9}^{-1 / 2} r^{-7 / 4} \operatorname{rad~m}{ }^{-2} .
$$

Equation (17) shows that large rotation measures and the associated depolarization of synchrotron emission by Faraday rotation are generic features of ADAF models (unless $\dot{m}_{-4} \ll 1$ ).

The absence of observed linear polarization in the radio spectrum of a low-luminosity galactic nucleus would be consistent with ADAF models. By contrast, detected linear polarization would argue against an ADAF as the source of the observed radio emission. A particularly interesting class of systems for future polarimetry are elliptical galaxies in nearby X-ray clusters (e.g., NGC 4649, 4472, and 4636 in the Virgo cluster). As discussed by, e.g., Fabian \& Canizares (1988), Fabian \& Rees (1995), and Di Matteo et al. (1999, 2000), many of these galaxies have extremely dim nuclei given the inferred black hole masses $\left(\sim 10^{9} M_{\odot}\right)$ and Bondi accretion rates. Linear polarization may shed important light on the physics of these systems.

We thank Don Backer, Roger Blandford, and Mark Reid for useful correspondence, Bruce Draine for useful conversations, and John Bahcall, Heino Falcke, and Feryal Özel for helpful comments on the paper. EQ is supported by NASA through Chandra Fellowship PF9-10008, awarded by the Chandra X-ray Center, which is operated by the Smithsonian Astrophysical Observatory for NASA under contract NAS 8-39073. AG was supported by the W. M. Keck Foundation and NSF PHY-9513835.

\footnotetext{
${ }^{7}$ For example, in Stone et al.'s (1999) simulations of non-radiating accretion flows, the density varies with polar angle roughly as $\rho \propto \sin ^{2} \theta$ (see also Quataert \& Gruzinov 2000); thus for $\dot{M} \sim \dot{M}_{\text {Bondi }}$, the emission must be confined to $\theta \lesssim 3^{\circ}$ in order for the density to be sufficiently small that high frequency emission is not depolarized. In addition our line of sight must lie within $\lesssim 3^{\circ}$ of the rotation axis of the flow.
} 


\section{A. Faraday rotation in an ultra-relativistic Maxwellian plasma}

Faraday rotation in a cold plasma is described by a change in position angle given by

$$
\frac{d \theta}{d l}=\frac{k_{\|}}{2} \frac{\omega_{p}^{2} \omega_{B}}{\omega^{3}}
$$

where $\omega=c k$ is the frequency of the radio wave, $k_{\|}$is the projection of the wavenumber along the magnetic field, $\omega_{p}^{2}=4 \pi n e^{2} / m_{e}$ is the plasma frequency, and $\omega_{B}=e B /\left(m_{e} c\right)$ is the cyclotron frequency. This corresponds to the usual rotation measure

$$
R M \equiv \frac{\theta}{\lambda^{2}}=\frac{e^{3}}{2 \pi m_{e}^{2} c^{4}} \int d \mathbf{l} \cdot \mathbf{B} n=2.63 \times 10^{-13} \times \int d \mathbf{l} \cdot \mathbf{B} n \frac{\mathrm{rad}}{\mathrm{m}^{2}} .
$$

Here we derive the rotation measure for an ultrarelativistic Maxwellian plasma:

$$
R M_{\gamma}=\frac{e^{3}}{2 \pi m_{e}^{2} c^{4}} \int d \mathbf{l} \cdot \mathbf{B} n \frac{\log \gamma}{2 \gamma^{2}}=2.63 \times 10^{-13} \times \int d \mathbf{l} \cdot \mathbf{B} n \frac{\log \gamma}{2 \gamma^{2}} \frac{\mathrm{rad}}{\mathrm{m}^{2}}
$$

where we have defined $\gamma \equiv k T_{e} /\left(m_{e} c^{2}\right)$. The dominant correction to the non-relativistic expression is the relativistic mass: $m_{e} \rightarrow \gamma m_{e}$.

We use the Vlasov equations to calculate the plasma permittivity and hence the dispersion

relation for electromagnetic waves. For a magnetic field and wavenumber along the $\mathrm{z}$ axis, the first-order (in the unperturbed magnetic field) permittivity is given by

$$
\epsilon_{x y}^{(1)}=\frac{-i}{2 \omega} \frac{4 \pi e^{2}}{m_{e}} \frac{e B}{m_{e} c} \int d^{3} p \frac{1}{\left(\omega-k v_{z}\right)^{2}} \frac{p_{\perp}^{2}}{p} \frac{d F}{d p} \frac{m_{e}^{2} c^{2}}{p^{2}+m_{e}^{2} c^{2}},
$$

where the unperturbed distribution function is normalized by $\int d^{3} p F(p)=n$, and $p_{\perp}^{2} \equiv p_{x}^{2}+p_{y}^{2}$. For a cold plasma, equation (A4) gives

$$
\epsilon_{x y}=\frac{i \omega_{p}^{2} \omega_{B}}{\omega^{3}}
$$

Using standard arguments (e.g., Rybicki \& Lightman 1979) this leads to the RM for a cold plasma given by equation (A2). For an ultra-relativistic plasma, equation (A4) gives

$$
\epsilon_{x y}=\frac{i \omega_{p}^{2} \omega_{B}}{\omega^{3}} \frac{\log \gamma}{2 \gamma^{2}}
$$

where we have not changed the definition of $\omega_{p}$ and $\omega_{B}$ in the ultra-relativistic regime. Equation (A6) for the permittivity gives the ultra-relativistic RM in equation (A3). 


\section{REFERENCES}

Abramowicz, M., Chen, X., Kato, S., Lasota, J.P., \& Regev, O., 1995, ApJ, 438, L37

Agol, E., ApJ Letters, in press (astro-ph/0005051)

Aitken, D.K. et al., 2000, ApJ, 534, L173

Beckert, T., \& Duschl, W. J. 1997, A\&A, 328, 95

Blandford, R.D. \& Begelman, M.C., 1999, MNRAS, 303, L1

Bower, G. C., Backer, D. C., Zhao, J., Miller, G., \& Falcke, H., 1999a, ApJ, 521, 582

Bower, G. C., Wright, M. C. H., Backer, D. C., \& Falcke, H., 1999b, ApJ, 527, 851

Burbidge, G. R. 1958, ApJ, 127, 48

Coker, R. \& Melia, F., 1997, ApJ, 488, L149

Di Matteo, T., Fabian, A. C., Rees, M. J., Carilli, C. L., \& Ivison, R. J., 1999, MNRAS, 305, 492

Di Matteo, T., Quataert, E., Allen, S., Narayan, R., \& Fabian, A. C., 2000, MNRAS, 311, 507

Fabian, A. C. \& Canizares, C. R., 1988, Nature, 333, 829

Fabian, A. C. \& Rees, M. J., 1995, MNRAS, 277, L55

Falcke, H., 1996, in Unsolved Problems of the Milky Way, eds. L. Blitz \& P. J. Teuben, IAU Symp. 169 , p. 163-175 (astro-ph/9411065)

Falcke, H., 1999, in The Central Parsecs of the Galaxy, eds. H. Falcke et al., ASP Conf. Series, Vol. 186, p. 148 (astro-ph/9909439)

Falcke, H., Mannheim, K., \& Biermann, P. L., 1993, A\&A, 278, L1

Ginzburg, V. L. \& Syrovatskii, S. I., 1969, ARA\&A, 7, 375

Gruzinov, A., 1999, in Proceedings IAU Symposium 195, in press astro-ph/9908027)

Igumenshchev, I.V. \& Abramowicz, M. A., 1999, MNRAS, 303, 309

Igumenshchev, I.V. \& Abramowicz, M. A., 2000, ApJ submitted astro-ph/0003397)

Igumenshchev, I.V., Abramowicz, M. A., \& Narayan, R., 2000, ApJ Letters submitted (astro$\mathrm{ph} / 0004006)$

Jones, T. W. \& O’Dell, S. L., 1977, ApJ, 214, 522 
Krichbaum, T. P. et al., 1998, A\&A, 335, L106

Lo, K. Y., Shen, Z.-Q., Zhao, J.-H., \& Ho, P.T.P., 1998, ApJ, 508, L61

Melia, F., 1992, ApJ, 387, L25

Melia, F., 1994, ApJ, 426, 577

Nagar, N. M., Falcke, H., Wilson, A., \& Ho, L. C., 2000, ApJ, in press (astro-ph/0005382)

Narayan, R., Igumenshchev, I. V., \& Abramowicz, M. A., 2000, ApJ in press astro-ph/9912449)

Narayan, R., Mahadevan, R., Grindlay, J.E., Popham, R.G., \& Gammie, C., 1998, ApJ, 492, 554

Narayan, R. \& Yi, I., 1994, ApJ, 428, L13

Narayan, R. \& Yi, I., 1995, ApJ, 444, 231

Narayan, R., Yi, I., \& Mahadevan, R., 1995, Nature, 374, 623

Özel, F., Psaltis, D., \& Narayan, R., 2000, ApJ in press (astro-ph/0004195)

Pacholczyk A. G., 1970, Radio Astrophysics (San Fransisco: W.H. Freemen \& Co.)

Quataert, E. \& Narayan, R., 1999, ApJ, 520, 298

Quataert, E., Narayan, R., \& Reid, M. J., 1999, ApJ, 517, L101

Quataert, E. \& Gruzinov, A., 2000, ApJ in press (astro-ph/9912440)

Rees, M. J., Begelman, M. C., Blandford, R. D., \& Phinney, E. S., 1982, Nature, 295, 17

Rybicki, G. \& Lightman, A., 1979, Radiative Processes in Astrophysics (New York: John Wiley \& Sons)

Serabyn, E., Carlstrom, J., Lay, O., Lis, D.C., Hunter, T.R., \& Lacy, J.H., 1997, ApJ, 490, L77

Stone, J. M., Pringle, J. E., \& Begelman, M. C., 1999, MNRAS, 310, 1002

Tribble, P. C., 1991, MNRAS, 250, 726

Tsuboi, M., Miyazaki, A., \& Tsutsumi, T., 1999, in The Central Parsecs of the Galaxy, eds. H. Falcke et al., ASP Conf. Series, Vol. 186, p. 105 